\title{
«Traduire ce que le poème fait à sa langue ». Entretien avec Martin Rueff par Mathilde Vischer
}

"Translate What the Poem Does to Your Language". Interview with Martin Rueff by Mathilde Vischer

\section{Martin Rueff et Mathilde Vischer}

\section{OpenEdition}

\section{Journals}

Édition électronique

URL : http://journals.openedition.org/recherchestravaux/1853

DOI : $10.4000 /$ recherchestravaux.1853

ISSN : 1969-6434

Éditeur

UGA Éditions/Université Grenoble Alpes

Édition imprimée

ISBN : 978-2-37747-165-2

ISSN : 0151-1874

Référence électronique

Martin Rueff et Mathilde Vischer, « «Traduire ce que le poème fait à sa langue ». Entretien avec Martin Rueff par Mathilde Vischer », Recherches \& Travaux [En ligne], 95 | 2019, mis en ligne le 05 décembre 2019, consulté le 08 septembre 2020. URL : http://journals.openedition.org/recherchestravaux/1853 ; DOI : https://doi.org/10.4000/recherchestravaux.1853

Ce document a été généré automatiquement le 8 septembre 2020

(c) Recherches \& Travaux 


\section{« Traduire ce que le poème fait à sa langue ». Entretien avec Martin Rueff par Mathilde Vischer}

"Translate What the Poem Does to Your Language". Interview with Martin Rueff by Mathilde Vischer

Martin Rueff et Mathilde Vischer

\section{NOTE DE L'ÉDITEUR}

Cet entretien a été conduit par écrit durant les mois de juillet et août 2018.

Mathilde Vischer: Martin Rueff, vous avez énormément traduit : de très nombreux poètes, des romanciers, des historiens et des philosophes italiens. À l'exception de Judith Butler (Qu'est-ce qu'une vie bonne?, Paris, Payot, 2014) et de David Graeber (Des fins du capitalisme, Possibilités 1, Paris, Payot, 2014), je crois, la plupart des auteurs que vous avez traduits le sont de l'italien. Pouvez-vous nous rappeler pourquoi avoir choisi cette langue, puisque rien ne vous destinait à elle a priori?

Martin Rueff : La formule du potache «je t'aime, c'est ma version » ne me distingue guère parmi les forts en thème de ma génération pour laquelle être un littéraire (description administrative qui correspondait au vœu le plus profond, imagerie de l'homme de lettres remisée au placard des antiquités), c'était d'abord et toujours traduire. Né au Canada anglais, mais lycéen à Marseille, je passais mes heures à traduire : du grec, de l'anglais, de l'allemand. Les langues familiales, hélas, perdues. J'y insiste : du grec surtout. Je ne crois pas appartenir à la dernière génération qui soit partie déchiffrer le Phèdre sous les pins. Et, aussi loin que je remonte, tout n'était que matin, éclats, promesses, scintilles, santé, fêtes, saillies, pulpe - vie aux soleils ruisselants et au goût d'aoristes. N'est-il pas étrange que les premiers verbes des langues anciennes qu'il nous était demandé d'apprendre fussent amo pour le latin et luo pour le grec, comme si, dans l'enseignement des langues mortes, et dans la forte influence de leur littérature qui ne manquerait d'en découler, nous avions été conviés 
à nous lier et à nous délier? C'est pourtant de luo qu'il faut parler d'abord. Luo : je délie. Verbe merveilleux - poésie des libertés ou des libertins selon qu'on défasse les chaînes de l'esclave, la tresse ou la ceinture de l'aimée! Trois lettres pour se détacher, c'est peu, mais que ce verbe soit d'emblée tout uniment positif et de déliaison, voilà qui est extraordinaire. Car pour nos langues analytiques, délier ne peut être que le mouvement second qui survient, dans la langue, après l'opération des liens : il en est ainsi en français, en italien (legare, slegare) et en anglais (tie, untie). Pour les Grecs, la déliaison est première dans la langue. Ne faudrait-il pas, aussi, avant d'en tirer une leçon de politique, conclure qu'il faut apprendre luo avant de connaître amo?

D'italien point. Mes études parisiennes ne m'y portèrent pas davantage. Et quand je dus choisir un poste de "volontaire du Service national» (VSN), j'élus le grand lointain: l'Inde. Mais le ministère en décida autrement. On cherchait un jeune agrégé. L'alternative ne fut guère amène: Bologne ou la caserne de Manosque. Je ne balançai pas et renonçai à New Dehli.

L'italien fut un enchantement et comme une création continue. La décision de traduire, je peux la faire remonter à un épisode précis: en Italie, le bachelier passe son grand oral devant une commission composée de tous les professeurs. Il me fallait donc entendre toutes les épreuves. La scène se passe à l'institut de la Santissima Annunziata, à Poggio Imperiale, un lycée au-dessus de Florence.

Un étudiant présente un poème de Montale qui remonte à 1916 et qui devait figurer dans les Ossi di seppia :

Meriggiare pallido e assorto

presso un rovente muro d'orto,

ascoltare tra i pruni e gli sterpi

schiocchi di merli, frusci di serpi ${ }^{1}$.

Montale n'a pas inventé le verbe « meriggiare » qui signifie « passer l'après-midi en se protégeant du soleil ». On trouve le verbe chez Gozzano ou chez D'Annunzio, mais à l'époque, la conviction qu'il s'agissait d'une invention poétique, et, à tout le moins la découverte qu'on pût utiliser un verbe synthétique là où le français recourt à une périphrase, tout cela, pardonnez-moi le ridicule, causa un émoi qui n’a pas cessé.

Cela dit, pour la revue Po\&sie (grande revue de traducteurs' ${ }^{2}$ ), j'ai traduit des articles de l'anglais, de l'allemand et des poèmes aussi. J'aimerais connaître d'autres langues. Mais l'italien a un privilège indéniable - il est aussi devenu une langue de vie et de travail. J'écris dans sa proximité exigeante.

Mathilde Vischer: Parmi les essais, on compte un nombre important d'ouvrages du philosophe Giorgio Agamben et de l'historien Carlo Ginzburg. Qu'est-ce qui a déterminé cette fidélité à leurs écrits?

Martin Rueff : Ne pourrait-on pas une fois pour toutes renverser l'adage «traduttore traditore " (qui révèle sa signification si on rappelle l'usage des traducteurs dans la diplomatie) et rappeler que la traduction est affaire de fidélité, et même de très haute-fidélité (ce qui explique aussi que des traducteurs veuillent capter des 
écrivains : fidèles à des créateurs ils voudraient que ces derniers leur jurassent, en retour, une fidélité éternelle, ce qui est mauvais)?

J'ai eu la chance d'être approché par des intellectuels italiens de premier plan qui m'ont confié la traduction de leur texte. Je leur suis resté fidèle. J'ai beaucoup appris d'eux. Je ne pourrais nouer ici un propos qui dépasse la vie privée des amitiés qu'à la condition de pointer chez le philosophe et l'historien un foyer que j'ai reconnu progressivement comme mien.

Agamben et Ginzburg sont, à leur manière, deux philologues. Agamben exploite philosophiquement les ressources de la philologie et philologiquement celles de la philosophie. Ginzburg a une connaissance historique de la philologie et explicite sa méthode dans un rapport étroit avec cette discipline dont il commente les grands maîtres. Philologie, philosophie et sciences humaines - cette poussée, aussi étonnant que cela puisse sembler, accompagne chez moi le poème dans une tension continue avec la traduction de l'italien.

Mathilde Vischer: Vous avez signé en 2018 la retraduction de la trilogie des ancêtres de Calvino (Le Vicomte pourfendu, Le Baron perché et Le Chevalier inexistant ${ }^{3}$ ) pour les éditions Gallimard. Si l'on compare votre traduction et celles de vos prédécesseurs, on est frappé, notamment, par le travail sur la syntaxe, beaucoup plus proche du rythme de la phrase italienne (je pense notamment à la répétition du « et », très fréquent en italien, et que les traducteurs ont tendance à éliminer). Pouvez-vous nous en dire un peu plus sur les enjeux stylistiques de cette retraduction?

Martin Rueff : a) Sans doute une bonne manière d'écrire l'histoire de la traduction poétique serait-elle d'étudier les retraductions de certains poèmes qui furent tels qu'ils ne cessèrent d'appeler, de demander et d'exiger qu'on les reprît; et, tout comme Claude Lévi-Strauss évoquait le potentiel de transformation des mythes, de même on pourrait évoquer le potentiel de traduction de certains poèmes ${ }^{4}$. Cette tâche relève d'une "critique des traductions » au sens dégagé par Berman dans un livre célèbre $^{5}$ où il compare plusieurs traductions du poème de John Donne, "Going to Bed ». Critiquer les traductions, ce n'est pas faire l'œuvre d'un maître d'école qui distribuerait les bons et les mauvais points, c'est, tout en déployant de nouvelles catégories d'analyse ("projet de traduction», "idéal de traduction», "horizon traductif »), ouvrir des pans entiers de l'histoire de la poésie, c'est-à-dire de la sensibilité (tout comme étudier les Annonciations ou les Dépositions de croix de la Renaissance, c'est montrer l'histoire de la peinture au travail). Dans la préface du volume qu'il a dirigé sur la retraduction ${ }^{6}$, Jean-Patrice Courtois insiste à juste titre : la retraduction est une affaire d'esthétique et de poétique générales sous le chef d'une philosophie de l'histoire.

b) Puis-je appliquer cette critique des traductions à mon propre travail de retraduction des textes de Calvino? Je peux à tout le moins offrir quelques indications ou suggestions. Un avertissement précautionneux les précède : à de rares exceptions près, les traductions antérieures de Calvino étaient excellentes, souvent passionnantes et portées par le texte. Elles avaient cependant vieilli au regard de notre propre horizon linguistique et traductif (esthétique et poétique mêlées). 
Le Baron perché par exemple s'inscrit dans l'horizon littéraire, politique et philosophique des Lumières. Calvino revendique cet héritage romanesque dans une note, il fait dialoguer Cosimo avec les philosophes, lui fait écrire à Diderot et imiter Rousseau. Le brigand pleure à la lecture de Richardson, le précepteur est emprisonné parce qu'il possède un exemplaire d'un texte de Bayle. Or cet héritage est stylistique aussi. La syntaxe du Baron n'est pas celle des autres romans de Calvino. La phrase est longue, articulée, sinueuse parfois ${ }^{7}$. Cette longueur permet des effets de rupture en fin de paragraphe - et il arrive souvent que Calvino termine un long développement par une phrase courte, comme le fait Voltaire par exemple. Inquiets peut-être de la lourdeur de style, mes prédécesseurs avaient coupé la phrase du Baron et changé son rythme, son allure. J'ai voulu rétablir la syntaxe de Calvino. Je n'ai pas craint en effet la répétition $\mathrm{du}$ " et ». Calvino est un écrivain économe, rapide - s'il répète il le fait à dessein. Et l'hyperbate n'est pas à craindre.

Pour ce qui est de Le Chevalier inexistant, il me semble que dans un livre qui porte pour l'essentiel sur ce que signifie exister ou "inexister" (je n'exclus pas que Calvino veuille aussi se moquer de l'existentialisme), on doit se rendre attentif à la grammaire du verbe "être» et du verbe "exister», et ne pas traduire l'un pour l'autre fût-ce risqué pour l'oreille. On ne peut pas traduire «c'è » et «non c'è » sans faire attention au sens du roman.

On peut ici penser à La Religieuse de Diderot. Dans un passage extraordinaire du roman, la religieuse remarque : « [...] il reste à savoir si ces actions sont de l'homme, et s'il y est, quoiqu'il paraisse y être ». "Paraître y être sans y être ", cette formule définit l'inexistence du chevalier. Elle implique des décisions du traducteur.

Mathilde Vischer : Dans un entretien pour Po\&sie paru en 2007, vous expliquez comment la poésie s'est toujours imposée à vous, tandis que la forme du roman « ne [vous] vient pas ${ }^{8}$ ». Cette impossibilité d'écrire des romans ne vous a pas interdit d'en traduire. L'écriture seconde romanesque n'a donc pas permis d'ouvrir à l'exploration de la forme du roman?

Martin Rueff : La retraduction des livres d'un romancier génial (qui se présentait plus volontiers comme un "narrateur ») a entraîné plusieurs conséquences dans mon travail d'écrivain. Elle a inspiré de nombreux poèmes. Il faudrait dire pourquoi. C'est sans doute parce que, pour bien traduire, il faut se mettre dans une disponibilité particulière par rapport à sa propre langue - quelque chose comme une «écoute flottante». On devient attentif à tel ou tel mot, à telle ou telle tournure. Calvino est une source d'inspiration, c'est certain.

Mais loin qu'elle ait diminué, la conviction que l'écriture du roman m'est complètement étrangère s'est renforcée. Je pourrais renverser la formule de Beckett qui répondait à Libération en 1981 à la question «Pourquoi écrivez-vous?», «Bon qu'à ça » et proposer « Pas bon à ça, mais alors pas bon du tout ».

Mais cela ne suffirait pas évidemment. Rien à voir cependant (la précision est superflue) avec je ne sais quelle rivalité avec le romancier - j'admire le roman (faut-il le préciser ?), je lis beaucoup de romans (anciens, modernes et contemporains) et je mets au plus haut certains romans (mes exemples ne brilleraient pas par leur originalité). Je puis dire qu'après avoir lu Claude Simon, je me suis dit que ce qu'avait 
réussi cet auteur dépassait de loin ce qu'un moderne pouvait rechercher dans le roman et son œuvre lue et relue reste devant moi. J'aime aussi qu'on me conseille des romans. J'ai la chance de fréquenter des romancières et des romanciers magnifiques. Pas de malentendu ici ni de procès d'intention.

C'est très simple et Calvino m'a confirmé dans cette conviction simple : je ne sais ou je ne veux pas raconter. C'est tout. C'est un rapport à la phrase, au temps impliqué dans la phrase - «non fa per me » comme on le dit en italien'.

Mathilde Vischer : Toujours dans ce même entretien, vous dites que votre histoire familiale aurait pu donner matière au romancier que vous n'êtes pas, et que votre "fidélité à cette histoire passe peut-être par sa négation dans le poème ». Pourtant, les quelques éléments que vous donnez (une partie de votre famille assassinée dans les camps, une autre exilée par la guerre d'Algérie, des abandons, des exils, pas de lieu «propre ») laissent à penser que ces expériences radicales, mêmes vécues à travers des récits, sont ce qui marque la langue de tout écrivain qui s'inscrit dans leur « après » (comme le montrent notamment les travaux de Frosa Pejoska-Bouchereau ${ }^{10}$ ). Percevez-vous un impact de ces événements dans la langue? Ou dans le fait d'être tellement à votre aise dans une activité qui est justement celle du «non-lieu », de l'étrangeté et de la différenciation (la traduction)?

Martin Rueff : Les travaux importants de Frosa Pejoska-Bouchereau portent sur les migrants obligés à l'exil par le présent et l'histoire. Ce n'est pas mon cas et mes exils comme mes éloignements relèvent du choix et de la chance aussi - ou de la transformation d'une bougeotte atavique parce que subie en une bougeotte d'élection. Mais ces migrations forcées et souvent mortelles furent le lot de mes arrière-grands-parents, de mes grands-parents et de mes parents aussi. Leur histoire est terrible et elle se distingue peu de celle des juifs d'Europe centrale arrivés en France d'un côté et de celle des Pieds-noirs de l'autre. Leur histoire est en partie mon histoire, mais la mythologie des vaincus n'est pas moins repoussante que celle des vainqueurs. Les héritiers ne sont pas plus comptables des horreurs subies par leurs aïeux que des horreurs qu'ils ont infligées. La mémoire privée envahit l'histoire et chacun se fait aujourd'hui une gloriole de son passé familial quand la culture nous apprend plutôt à nous méfier. Il faut ici comme ailleurs vivre selon la profonde invitation de Pascal quand il met en scène son naufragé « avec une double pensée ».

Pour ce qui me concerne, si je me penchais sur la question, je devrais faire la part de ce à quoi je m'arrache et qui me reste attaché par cet effort même. Mon penchant pour la traduction est aussi (et peut-être d'abord) lié à des lectures, à des goûts, à une histoire intellectuelle et à un amour qui a des racines très profondes (qu'on pourrait peut-être rapporter à une histoire qui me précède) : celui de la langue. L'amour, la langue, la poésie. Ce triangle fonctionne pour moi dans tous les sens et s'il repose sur des expériences très dures, il est porteur de joie.

Mathilde Vischer: La traduction de la poésie serait, d'après vos propres termes, une « pratique de désubjectivation ». Comment le nœud subjectivation-langage se manifeste-t-il 
au sein du poème traduit ? En quoi la traduction de la poésie est-elle pour vous également une pratique théorique?

Martin Rueff : a) En dépit d'efforts anciens, souvent maladroits, et parfois excitants quand ils ne relèvent pas du truisme, la pensée du lyrisme attend son lieu d'élaboration théorique générale. Il me semble cependant (il est difficile ici de simplifier) que ce que cherchent à élaborer de nombreux courants philosophiques contemporains tourne autour de la définition d'individuations non subjectives (que ce soit la phénoménologie, ou la pensée wittgensteinienne quand elle cherche à comprendre ce que signifie dire "je » et on pourrait même évoquer, avec bien des précautions, Foucault quand il évoque les " processus de subjectivation ${ }^{11}$ » ou Deleuze dans son article stimulant "Réponse à une question sur le sujet ${ }^{12}$ » en réponse à une question de Jean-Luc Nancy).

Or à bien considérer l'histoire du genre, on peut constater que la poésie a sur ce terrain des longueurs d'avance. Il suffit de lire attentivement Les Métamorphoses d'Ovide. Leur incipit n'est-il pas source d'émerveillement: «In nova fert animus mutatas dicere formas / corpora ${ }^{13}$ » ? La construction de la différence phrase / vers, le rejet de "corpora » au second vers - tout cela est l'affaire d'un génie de la langue. Mais il ressort clairement que ce que traque Ovide, ce ne sont pas des corps qui changent de forme (des corps si l'on peut dire comme avides de leur identité transie), mais des formes qui traversent des corps. Ovide, entre plusieurs exemples bien sûr mais la désubjectivation subjectivante est partout dans la poésie et les exemples sont si nombreux dans la poésie des $\mathrm{XIX}^{\mathrm{e}}$ et $\mathrm{XX}^{\mathrm{e}}$ siècles qu'il faudrait réécrire son histoire en recourant à cette hypothèse.

On pourrait citer la lettre de Keats à Woodhouse du 27 octobre 1818. Keats explique sans ambages que le je poétique n'est pas un je, il n'est pas identique à soi: "En ce qui concerne le caractère poétique lui-même (j'entends de l'espèce à laquelle, pour autant que je relève d'aucune, j'appartiens ; [...]) il n'est pas lui-même, - il n'a pas de moi - il est tout et rien - il n'a pas de caractère (it is not itself - it has no self - it is everything and nothing - it has no character $)^{14} »$.

$\mathrm{Au} \mathrm{xx}^{\mathrm{e}}$ siècle, on peut dire que certains poètes se sont persuadés que la traduction pouvait être une des cordes de leur lyre impersonnelle : par la traduction, le poète fait l'épreuve des transactions délicates et douloureuses qu'implique la vie de tout sujet dans une langue (puisqu'il est déjà délicat d'écrire dans sa langue) comme votre très bel ouvrage le montre ${ }^{15}$. Bernard Simeone l'écrit fort nettement: «traduire désapprend la possession, l'identification, l'idolâtrie toujours aux aguets dans le rapport à ce qui s'inscrit ${ }^{16} »$. On peut le dire autrement et de manière plus technique : s'il est difficile de comprendre ce qui se passe quand un écrivain en vers écrit «je » dans un poème, il est encore plus difficile de comprendre ce qui se passe quand un autre écrivain en vers traduit «I », « Io », « Ich » par « Je ».

b) Quant à la question de savoir en quoi la traduction est une " pratique théorique ", elle vient de mes travaux sur Althusser et sur Foucault. C'est dans un des textes de son Pour Marx ${ }^{17}$ qu'Althusser essaie de dépasser l'opposition de la pratique et de la théorie (la version classique de cette opposition est exposée de manière définitive par Kant dans un opuscule de $1793^{18}$ ). Althusser voulait faire entendre au P.C.F. une thèse importante : Marx est un philosophe et en tant que philosophe il peut servir la lutte. Pour que cela fût audible, il fallait démontrer que la philosophie comme théorie avait 
une portée pratique - que la philosophie pouvait se présenter comme une pratique théorique et pas seulement comme une théorie de la pratique. Je me suis donc demandé si ce débat ne pouvait pas être transposé dans le champ de bataille de la traductologie.

Quand il s'agit de traduction de poésie, on est souvent confronté à une alternative sommaire et assommante : d'une part les spontanéistes ( « je traduis le poème comme je le sens... », «il n'y a pas de règles... ») et les traductologues toujours prêts à émettre des lois générales et des théories échevelées que le traducteur a du mal à appliquer quand il se trouve au pied du texte. Il se trouve que l'argument d'Althusser peut aider à réfléchir sur cette alternative. Comparer des traductions, par exemple, permet de rendre évidents des choix de traduction et il n'est pas très difficile de montrer en quoi ces choix relèvent d'une théorie, fût-elle minimale. La traduction rend vivant notre sentiment de la langue et ce sentiment dicte souvent des options lourdes qui forment des théories sous-jacentes d'autant plus fortes qu'elles sont aveugles à elles-mêmes.

Mathilde Vischer : Pour décrire la traduction, vous avez dit lors d'une conférence une chose qui me semble très juste: il s'agit de «refaire dans sa langue ce que le poème fait à la langue de départ ${ }^{19} »$. Cette façon d'évoquer l'acte de traduire nous ramène à la question centrale de la singularité subversive de l'écriture poétique et aussi à celle du rythme, peutêtre la notion la plus importante et la plus délicate en traduction. De façon tout à fait concrète, comment procédez-vous pour tenter de retrouver, à chaque fois de façon singulière, le geste du poème, sa respiration, et aussi, parfois, sa dimension subversive face au langage?

Martin Rueff : Ce qui est horriblement difficile, ce n'est pas de traduire ce que fait le poème dans la langue de départ (même si ce premier niveau n'a rien d'aisé - il faut une connaissance qui tient autant de l'encyclopédie que du dictionnaire : les pièges sont partout et les glissades à chaque pas), mais ce que fait le poème à la langue.

Les exemples sont nombreux de très bonnes traductions qui ratent cet effet - ce ratage s'explique par ce qu'il faut appeler un véritable obstacle épistémologique qui se dresse face à tout traducteur de poèmes qui traduit avec ce qu'il croit être « de la » poésie, relever "de la poésie ", "être poétique ». C'est aussi le cas du lecteur dont l'horizon d'attente en poésie est si bien défini que Renan, préfaçant sa traduction du Cantique des cantiques, prévient que seront « choquées les personnes qui n'admirent de l'Antiquité que ce qui ressemble plus ou moins aux formes du goût français ${ }^{20}$ ».

Or traduire un poète, c'est essayer de se mesurer avec une idée inédite de la poésie qui oblige à se défaire des préjugés. On trouve à ce sujet une belle anecdote dans Le Jardin des Finzi-Contini $i^{21}$, le grand roman de Bassani. Les deux protagonistes rédigent l'un et l'autre des mémoires sur des poètes - tout comme le narrateur est inscrit en tesi di laurea à Bologne sur Panzacchi, Micòl est inscrite à Venise sur la poétesse Emily Dickinson : « pour le choisir [mon sujet de diplôme], je l'ai choisi ; Emily Dickinson, tu sais bien, cette poétesse américaine, cette espèce de femme terrible... » C'est dans cette situation de symétrie que le narrateur reçoit de Venise une étrange lettre de Micòl. "C'était une petite lettre spirituelle, ni trop longue ni trop brève, écrite au recto et au verso de deux feuilles de papier bleu clair qu'une calligraphie à la fois impétueuse et légère avait prestement remplie, sans hésitations ni ratures. »Cette 
lettre de Micòl se «termine inopinément (a sorpresa) par la traduction en vers d'un poème d'Emily Dickinson ». Bassani cite alors l'intégralité du poème :

\begin{tabular}{|c|c|}
\hline $\begin{array}{l}\text { Morii per la Bellezza; e da poco ero } \\
\text { discesa nell'avello, } \\
\text { che, caduto per il Vero, uno fu messo } \\
\text { nell'attiguo sacello. }\end{array}$ & $\begin{array}{l}\text { Je suis morte pour la beauté ; et j'étais depuis peu } \\
\text { descendue au sépulcre, } \\
\text { quand tombé pour le Vrai, quelqu'un fut mis } \\
\text { dans le sacellum contigu. }\end{array}$ \\
\hline $\begin{array}{l}\text { «Perché sei morta? " mi chiese } \\
\text { sommesso. } \\
\text { Dissi : « Morii pel Bello ». } \\
\text { « Io per la Verità : dunque è lo stesso, } \\
\text { - disse - , son tuo fratello.» }\end{array}$ & $\begin{array}{l}\text { «Pourquoi es-tu morte? ? me demanda-t-il à mi- } \\
\text { voix. } \\
\text { Je répondis : «Je suis morte pour le Beau ». } \\
\text { « Moi pour la Vérité : donc, c'est la même chose, } \\
\text { dit-il-, je suis ton frère. » }\end{array}$ \\
\hline $\begin{array}{l}\text { Da tomba a tomba, come due congiunti } \\
\text { incontrastisi a notte, } \\
\text { parlavamo così : finché raggiunti } \\
\text { l'erba ebbe nommi e bocche }{ }^{22} \text {. }\end{array}$ & $\begin{array}{l}\text { De tombe à tombe, comme deux parents } \\
\text { se rencontrant la nuit, } \\
\text { nous parlions ainsi ; jusqu'à ce qu'atteints } \\
\text { l'herbe prît les noms et les bouches }{ }^{23} \text {. }\end{array}$ \\
\hline
\end{tabular}

On peut faire l'hypothèse que le poème figure dans la narration pour dire une vérité qui ne se dirait pas sans lui. Plutôt que de l'interpréter, soulignons que ce texte constitue pour les protagonistes eux-mêmes un objet herméneutique. Micòl le commente ainsi dans le post-scriptum: "Alas poor Emily. Voilà le genre de compensation sur lequel l'abject célibat féminin est contraint de miser ». Et le narrateur se demande immédiatement si cette phrase vaut davantage pour Micòl que pour Emily - « une Micòl en phase dépressive, en phase d'autocommisération ». Dans sa réponse, le narrateur «s'en tient à la littérature ». Il précise qu'il loue le poème et sa traduction, beaux l'un comme l'autre. Il en commente la qualité et la «douceur carducienne » : mais ce compliment n'est pas simple.

C'est que traduire Dickinson dans la langue de Carducci est une captation esthétique qui dit bien l'enjeu de la traduction des poèmes. À l'école, Micòl s'est formée sur Dante et sur Carducci - quand elle doit affronter Dickinson elle ne peut faire autrement que rapporter ses vers à la musique poétique qu'elle a en tête : celle de Carducci.

De la même manière, le grand traducteur Gilles de Van avait traduit Travailler fatigue $^{24}$, le chef d'œuvre de Cesare Pavese, en accomplissant une opération analogue. On sait que Pavese invente en italien un vers long inspiré de Whitman qu'il avait étudié pour son mémoire de fin d'études soutenu grâce au truchement généreux de Leone Ginzburg. Traduire Pavese en français, c'est chercher un vers long, or De Van veut un vers plus court inspiré de Baudelaire et de Verlaine. Cela permet de faire passer Pavese pour un poète reconnaissable parce que son rythme ressemble à des chants anciens, mais on ne comprend pas alors ce que Pavese avait fait à la langue poétique italienne.

La preuve n'est-elle pas faite que la traduction relève de l'esthétique et de la poétique avant toute chose? Pour traduire un poète, il faut comprendre ce qu'il fait à sa langue - son opération poétique. 
Mathilde Vischer : Dans un texte portant sur l'écriture et la traduction de la poésie publié en 2013 en traduction italienne dans la revue Testo a fronte, vous écrivez «la poesia è quella pratica della lingua dove il ritmo delle parole è la forma dell'esperienza ${ }^{25}$ » [la poésie est cette pratique de la langue où le rythme des mots est la forme de l'expérience]. Qu'entendez-vous par « expérience » ici ?

Martin Rueff : Dans son Kantbuch, Heidegger souligne que chez Kant les conditions du sujet de l'expérience sont les conditions de l'expérience du sujet - la formule est difficile mais frappante. En d'autres termes, on ne saurait avoir d'expérience que dans la mesure où nous avons la possibilité de faire telle ou telle expérience. Ce que le poème met en évidence, c'est précisément que parmi ces conditions de l'expérience, il y a la langue. Notre expérience est tramée par les mots qui affluent (le mot est chez Michel Deguy) quand nous la traversons. Dans le poème je suis touché par une expérience en langue - quand la langue sert à schématiser l'expérience, le poème a gagné. Je veux décrire une course sur le sable, je pense immédiatement aux vers de Valéry :

Le frais gravier, qu'arrose et fuit sa course agile,

Croule, creuse rumeur de soif, et le facile

Sable a bu les baisers de ses bonds puérils [... ${ }^{26}$

Montale, Sereni configurent mon expérience de l'Italie. Mais que dire des objectivistes? Si nous tenons aux vers, si nous les citons, c'est parce qu'ils contiennent une part de notre expérience en langue qui est la langue de notre expérience. Mon attachement à la poésie et à la poétique de Michel Deguy tient en grande partie à cette conviction - il ne saurait y avoir de phénoménologie poétique sans prendre en compte la puissance phénoménale du logos poétique. Il faut méditer et méditer encore La poésie n'est pas seule : Court traité de poétique ${ }^{27}$.

Mathilde Vischer: La poésie italienne est par essence plurilingue, tandis que la poésie française, son histoire, est résistante à l'entremêlement des langues (cela change, cependant). Trois de vos «livres de poèmes » le font pourtant, et le quatrième est écrit entièrement en italien. Est-ce la longue fréquentation des écrivains italiens qui a rendu ce plurilinguisme et ce passage d'une langue à l'autre possibles? Si l'on compare les trois recueils de poèmes écrits en français, c'est dans Comme si quelque (2006) que le plurilinguisme est le plus présent: des sections entières sont en italien (et certains passages en anglais, d'autres fragments plus brefs en latin ou en allemand), l'incursion dans d'autres langues étant plus rare dans Lapidaire adolescent (2001) et Icare crie dans un ciel de craie (2006). Pouvez-vous nous dire ce qui détermine la nécessité de l'autre langue au cours d'un projet poétique?

Martin Rueff : J'avais tenu à ce que figurât dans l'anthologie de la poésie italienne proposée par la revue Po\&sie, « 30 ans de poésie italienne, 1975-2004²8 ", le texte magistral de Contini, « Preliminari sulla lingua del Petrarca » (1951). En opposant au plurilinguisme de Dante ${ }^{29}$ le monolinguisme de Pétrarque, Contini opposait bien deux poétiques. Deux styles d'abord : la tendance à embrasser la richesse du monde et de la langue d'une part (c'est elle qui fait la copia de la Comédie), la tendance à les concentrer dans une expérience, unitaire et rassembleuse (osera-t-on ? celle d'un Je transcendantal) de l'autre. Contini distinguait ainsi deux tendances dont il acceptait certes qu'elles pussent se ramener à des pôles dialectiques (Jakobson aurait dit des dominantes), mais qui formaient bien pour lui les deux extrémités d'un arc de cercle. En second lieu, la pluralité des tons et des niveaux de langue de Dante contre l'unité 
de tons, comme nappés chez Pétrarque. Troisième critère : l'opposition d'une poésie qui accepte les risques de la poétique et de la théorie (Dante) dans le poème contre une poésie de la spontanéité qui ne peut les accepter. Dernier critère enfin, l'opposition de l'expérimentation à tout crin et de son refus. Ces oppositions ne valent pas strictement pour départager Dante et Pétrarque - elles doivent être dialectisées pour devenir des catégories historiques descriptives.

Il reste que depuis la chasse menée par Boileau aux pétrarquistes, le poème français s'écrit en apparence en français dans la mesure où il exploite le « chant profond » de la langue - son rythme propre, ses cadences - le vers de Baudelaire frappe à jamais par le rythme qu'il donne à nos expériences, et il en va de même avec Apollinaire, mais aussi bien sûr avec Aragon - sa Diane est française. Le symbolisme ne touche pas à ce monolinguisme ni l'hermétisme. Saint-John Perse, Char, Dupin, Bonnefoy, Jaccottet sont en ce sens des poètes monolingues. Cet énoncé n'est pourtant pas tout à fait exact - car les poètes français sont souvent marqués par des langues qu'ils ont appris à traduire - vers latins de Rimbaud, legs de Poe chez Baudelaire et Mallarmé. Depuis Michel Deguy (encore lui), les poètes contemporains admettent davantage leur plurilinguisme (on peut penser par exemple à l'anglais chez Emmanuel Hocquard).

Dans mon cas, c'est le séjour en Italie qui a libéré les poèmes en français et, en même temps, les poèmes en italien. Ce voisinage correspond à toutes sortes de pratiques d'écriture, mais rarement à l'autotraduction, sauf dans un cas, dramatique - Corda tesa. Dramatique car je me suis traduit avec l'aide d'un ami traducteur italien qui venait de perdre un amour (une femme merveilleuse qui traduisait de l'italien au français). Or ce traducteur, Alessandro Serra, avec qui j'ai cotraduit le livre de Jacques Rancière sur Mallarmé ${ }^{30}$ est mort à son tour et la traduction de ce poème est contemporaine de sa maladie. Je me méfie toujours de la valeur prémonitoire des poèmes - ce sont les fameuses prophéties de d'Aubigné que Samuel Junod a étudiées dans un livre qui n'a pas reçu toute la reconnaissance qu'il mérite ${ }^{31}$.

L'autotraduction est jouée, et même surjouée et parodiée dans Icare crie dans un ciel de craie, livre dans lequel le rapport au plurilinguisme est le mieux explicité. Icare le fils européen, cloué sur son lit de corail est traversé par des poèmes anciens de toutes langues (le latin d'Ovide, l'allemand de Rilke, l'italien de Dante et d'Ungaretti, mais aussi l'anglais de Shakespeare et de W. Stevens). Il n'est pas particulièrement doué pour les langues, mais il est doté par elles. Les poèmes s'échappent de lui. Il ne peut les retenir. Ce sont un peu les dragées mirifiques de Rabelais.

J'avais décrit cela dans un court texte qui précédait des poèmes italiens dans Comme si quelque (je ne sais pas si je connaissais alors les poèmes valaisans de Rilke et les poèmes français d'Ungaretti, j'en doute en fait) : « Il est des moments où l'immersion dans une langue et dans l'œuvre de ses poètes, la fréquentation de leurs plus beaux vers et de leur musique, ainsi que la contemplation des paysages de l'arrière-pays entraînent chez l'étranger un effet comme hypnotique. Alors se lève en lui, dans une évidence pure parce qu'imposée, un poème non traduit. La leçon est sèche : quelle que soit la théorie qu'on en propose, la poésie est cette pratique de la langue où le rythme des mots est la forme de l'expérience ${ }^{32} »$. La question taraude de savoir pourquoi cette densité s'impose dans une langue et non dans une autre. La vibration que l'étranger ressent à tracer tel vers dans une langue qui n'est pas la sienne a 
quelque chose d'inexplicable. Cette évidence doit beaucoup aux poètes. (C'est pourquoi on trouve dans ces vers une série d'emprunts, indiqués en italique - saluons la justesse du terme - ramenés de coups de filets lents chez des auteurs chéris jusqu'aux larmes: Montale, Sereni, Pasolini). En 1967, Karl Shaeffer, un ancien commandant de U Boot de la Kriegsmarine, devenu après la guerre chef de l'entraînement de la marine américaine mettait en évidence sur deux plongeurs apnéistes l'effet de blood shift (ou "vasoconstriction périphérique ") déjà observé chez les dauphins. La lecture de la poésie italienne correspond, pour l'apnéiste que ne manque pas d'être l'étranger, au double effet du blood shift: protection vitale pour résister à la pression du monde et appel impérieux des profondeurs ${ }^{33}$.

Se laisser aller à la langue : je prends un exemple parmi ces poèmes de la section italienne de Comme si quelque - le sixième s'intitule «A forza di fare il morto ». C'est un poème de baignade nocturne inspiré par Dante, mais surtout par l'expression italienne «fare il morto" qui veut dire faire la planche - se laisser porter comme l'alcyon de Chénier ou le bouchon des stoïciens. La langue est pleine de catachrèses l'écriture d'un apprenti bilingue peut servir à réactiver les catachrèses à la manière dont les poéticiens parlent de réactivation étymologique.

Cela me pousse en retour à considérer souvent la langue française sous l'œil rêveur de la réactivation des catachrèses.

Mathilde Vischer: Vous avez traduit de très nombreux poètes de langue italienne, et dont les styles sont nécessairement très différents. II paraît donc difficile, comme on peut le faire pour des poètes-traducteurs qui sont restés fidèles à un même auteur, de déceler une influence d'un auteur précis sur votre travail poétique. Pensez-vous cependant que l'un ou l'autre des auteurs que vous avez traduits a eu une importance particulière pour ce que vous écrivez aujourd'hui ?

Martin Rueff : Les poètes traduits sont les « alliés substantiels » de Recherche de la base et du sommet ${ }^{34}$. On ne peut pas les imiter - ils montrent comment faire. Aucun écrivain en vers ne se lancerait dans ce métier d'écrire des vers s'il n'y avait des prédécesseurs qui avaient ouvert la voie en aérant la pièce. Comme le montre Kant, la valeur de l'inimitable et de l'original, la génialité se mesurent à leur contraire : à la possibilité de l'imitation et de l'itération. Le génie, écrit-il, est "musterhaft»: il constitue un exemple (Beispiel) qui se prête, non à l'imitation servile (Nachahmung), mais à être suivi (Nachfolge) et qui peut être simplement singé (Nachäffung), comme par un élève qui en imiterait aussi les défauts ${ }^{35}$.

Je pourrais alors imaginer une reconnaissance de dettes sur le modèle de celle pratiquée par Marc Aurèle - ce que je dois à... : à Zanzotto (la conviction du poème arche) qui embarque tout dans la langue, c'est une forme de force folle qui inspire confiance ; à De Signoribus (la force de la faiblesse - la résistance par la douceur), à Cecchinel (un rapport de double fidélité à l'histoire et à la métrique). Mais il faudrait immédiatement rappeler la part de poètes que je n'ai pas encore traduits et que je ne traduirai peut-être jamais et qui sont toujours là présents, lus, relus - et pour me limiter au domaine italien, Ungaretti, Montale, Sereni, Caproni. Les poètes que l'on traduit ne sont pas forcément ceux avec lesquels on écrit. 
Mathilde Vischer : Par l'exemple d'Artaud amené dans votre contribution au catalogue paru en marge de l'exposition sur la traduction Après Babel, traduire, vous mettez en évidence l'importance de la fragilité (être «mal armés6») pour traduire et pour écrire. La force de la traduction tiendrait-elle en sa capacité à nous révéler la vulnérabilité du texte et de la langue, et notre propre vulnérabilité face au texte et à la langue?

Martin Rueff : a) Artaud traducteur? Artaud figure défigurée du traducteur? On croirait à une provocation. Que valent les petites tentatives faites à Rodez entre 1943 et 1946 au regard de l'une des plus intenses, et des plus bouleversantes entreprises littéraires $\mathrm{du} \mathrm{xx}^{\mathrm{e}}$ siècle ? Le compte est vite fait : une vingtaine de pages, tout au plus, au regard des vingt-huit volumes de ses œuvres complètes. Pire, Artaud connaissait mal l'anglais («fort mal et pour ainsi dire pas du tout ${ }^{37}$ » écrit-il à Anaïs Nin en 1933) et n'aimait ni la traduction, ni l'adaptation («avec toutes les sales privautés que ce mot suppose avec un texte ${ }^{38}$ » écrit-il à propos de son « édition » du Moine de Lewis). C'est à Rodez où il se trouve interné du 11 février 1943 au 25 mai 1946 au terme d'un épouvantable périple commencé en Irlande à la fin du mois de septembre 1937, qu'Artaud entreprend la traduction de plusieurs textes anglais. À l'initiative du docteur Ferdière, psychiatre adepte de l'art-thérapie puis de l'abbé Henry Julien, angliciste et aumônier à Rodez de janvier 1941 à octobre 1944, Artaud va mener à bien au moins cinq adaptations de textes anglais. Il s'agit d'abord de trois adaptations de Lewis Carroll composées à la fin de l'été 1943 : « Variations à propos d'un thème ", d'après "Themes with variations ", "Le chevalier mate-tapis » d'après "Ye Carpette Knyghte "; "L'arve et l'aume ", d'après le chapitre VI de "Through the Looking Glass ». Par la suite il entreprendra la traduction de «Le bébé de feu » de Robert Southwell qui paraîtra en revue, et du poème d'Edgar Poe, «Israfel ». À ces cinq tentatives s'ajoute, semble-t-il, une traduction de «L'Ode au Rossignol » de Keats, et surtout de plusieurs autres poèmes de Poe, dont « Ulalume » et « Annabel Lee ». Paule Thévenin rapporte qu'Artaud aurait brûlé d'autres traductions de Poe parce que leur tonalité mystique ne lui convenait plus.

Or dans la traduction, Artaud cherche une voie pour l'insubordination - il dynamite l'original, fait sauter tous les gonds et ses traductions continuent à claquer comme une porte ouverte par le vent de son génie. L'étude systématique de ce dynamitage fut une entreprise passionnante.

b) Quant au rapport force/faiblesse, il est pour moi sans équivoque. En dépit de la lettre VII dans laquelle Platon condamne l'asthénie du langage, toute la force est du côté de la langue, de ses ressources, de son histoire, de son infinie beauté et toute la faiblesse du côté du traducteur comme de l'écrivain. Si toute traduction est un acte d'orgueil, son résultat invite à la modestie. La langue est le manteau, le poète, le démantelé.

Mathilde Vischer: Dans Icare crie dans un ciel de craie, que vous qualifiez de livre sur la filiation, le lecteur prend part à la chute d'Icare de la hauteur du soleil jusqu'au tréfonds de la mer, dans un rythme qui tient à la fois de l'écoulement continu et du souffle retenu. Je me disais que la plongée d'Icare pourrait peut-être aussi être lue comme la traversée du traducteur dans l'œuvre et la langue de l'autre : il traverse la tradition de sa propre langue (qui le traverse aussi), se confronte à ses pères et, avec Celan qui ouvre et clôt le livre, il se 
retrouve dans sa chute, «le ciel en abîme sous lui » (Le Méridien ${ }^{39}$ ), dans le renversement de la langue et de l'identité bouleversées.

Martin Rueff : Votre hypothèse, riche et inattendue, porte la marque de Baudelaire "plonger au fond du gouffre pour trouver du nouveau ». Plonger dans les langues pour trouver la langue des langues. Elle inspire plusieurs pensées dont la plus sombre: Icare se suicide et il est vrai que toute traduction comporte une part de drame et que le ratage est de rigueur. Cette part m'apparait moins grande cependant que dans l'entreprise du poème. Les poèmes résistent aux mauvais traducteurs (ils peuvent même sortir grandis d'une mauvaise traduction), les mauvais poèmes sont des plongées contre-productives - on pourrait risquer des « contre-plongées ».

Mathilde Vischer: Dans le double numéro de Po\&sie dédié à «30 ans de poésie italienne » que vous avez coordonné, cinq questions ont été posées aux poètes contemporains. J'aurais envie de vous les poser toutes, mais je me limiterai à une seule: "pourriez-vous donner une définition du langage poétique, et sinon, pourquoi »?

Martin Rueff : a) En mourant, Benveniste laisse un ensemble de fiches destinées à un livre sur Baudelaire. On vient de les éditer ${ }^{40}$. Benveniste se demande à plusieurs reprises si la poésie est une langue particulière, une langue détachée de la langue, une langue à part. La réponse est positive selon Benveniste qui ne convainc pas. Elle est pour moi négative. La poésie exploite le langage dans ses plus grandes possibilités, mais elle n'est pas un « langage » à part, une autre langue. Elle est un art du langage, une technique au matériau partagé. L'objection adressée à Mallarmé ne tient donc pas. On la trouve dans une note d'inspection d'un certain Lerambert: Mallarmé écrirait anglais en français ${ }^{41}$. Or on objecterait volontiers à l'inspecteur que si on peut écrire anglais en français (ou latin ou allemand), c'est parce que le français permet qu'on l'élargisse, qu'on augmente son élasticité comme le dit Chateaubriand quand il traduit Milton.

Le langage poétique comme ouvrage d'art oblige à un certain type de lecture - lente, rapprochée, attentive - comme lorsqu'on se penche sur un visage aimé. Pour lire un poète, il faut être prêt à se laisser déloger par ce travail de la perception imposé par ses inventions formelles. C'est la leçon de Proust - on apprend à aimer un visage (la temporalité est ici inverse du coup de foudre ou plutôt, aimer c'est apprendre à approfondir le coup de foudre dont la brûlure, loin de "passer », se fait plus mordante. Il en va de même quand on écoute en boucle un morceau de musique - au départ on pense qu'on va se l'approprier et puis progressivement c'est lui qui s'empare de vous).

Ce genre de travail est rendu possible par l'enseignement de la poésie, qu'il ne faut pas se hâter de condamner.

Comme d'autres j'imagine, je suis frappé par la différence de traitement entre les arts. Les peintres $d u x^{e}$ siècle ont réussi à modifier la perception du public. Les musiciens $\mathrm{du} \mathrm{xx}^{\mathrm{e}}$ siècle ont eux aussi réussi à modifier la perception du public. Mais dans le cas des poètes, on en reste à une espèce de "touche pas à ma langue " - ce qui explique aussi le soupçon ou la haine qui frappe la poésie. Poursuivons le parallèle : quand bien même ledit public ne serait pas habitué des vernissages d'un artiste à la mode, il sait que l'impressionnisme a vécu, que c'est un art qui a fait son temps. Il a 
l'idée que quelque chose s'est passé depuis, que l'on ne peint plus comme cela ou que, si on le fait, on peint "comme avant ». Le peintre du dimanche qui égaye son salon par une toile de fleurs pâles n'est pas un mauvais peintre - mais la formation de notre regard nous dit qu'il n'est pas notre contemporain. C'est ce que Hegel appelle, sans le moindre pathos apocalyptique, la «mort de l'art»-la conviction que la création artistique a une histoire et que se faire historien de l'art, c'est se demander pourquoi telle ou telle forme est possible à un moment de l'histoire, impossible à d'autres. Il faudrait peut-être dire - pourquoi un artiste rend telle ou telle forme possible à une époque.

Or si l'on considère la poésie, on est frappé de constater que le public de la poésie a un goût marqué pour les anciennes formes et que tout ce qui ne ressemble pas de près ou de loin à du Baudelaire ou à des chants doux amers à la manière d'Apollinaire est mal reconnu. Ce décalage explique en partie le désintérêt de notre société pour la poésie en train de se faire - et la question n'est pas de défendre telle ou telle forme de poésie et de se plaindre que l'avant-garde n'est pas suivie, que l'hermétisme n'est pas compris ou je ne sais quoi.

Seule une approche dialectique de la relation entre culture et langue peut expliquer le désamour du public français pour ses poètes dans une phase passionnante lors de laquelle de grands poètes, femmes et hommes, se sont déclarés.

Ce constat ne change rien au travail qui gagne à l'oublier. En revanche une question se pose - la poésie critique doit-elle faire état dans le poème de la situation de la poésie ou doit-elle réserver ses positions à la poétique ? La réponse à cette question départage nos contemporains.

Mathilde Vischer: Dans l'entretien pour Po\&sie paru en 2007 déjà cité, vous expliquez que l'un des malentendus concernant la perception de la poésie et des poètes réside dans le décalage entre une certaine image idéologique du poète et ce que le poète fait, qui semble mis à l'écart par ceux qui parlent de poésie. Vous insistez sur la nécessité d'une critique qui mette justement en valeur le travail des poètes. Onze ans après, pensez-vous que la situation ait changé?

Martin Rueff : Je craignais qu'elle n'empirât et elle a empiré. Le discours sur la poésie s'est raréfié, la description technique des opérations poétiques est devenue l'affaire d'une poignée qui se compte, comme les vers, sur les doigts de la main. Ce genre de constat doit être affiné sous peine de passer pour la plainte corporatiste d'un grincheux.

Pour instruire la question, il faut se demander sans tergiverser qui parle de poésie et pour dire quoi. Les journaux n'en parlent pas ou peu. Il a fallu l'extraordinaire publication des Euvres complètes de Mandelstam ${ }^{42}$ ou la mort d'Yves Bonnefoy pour qu'on parle de poésie dans la presse nationale.

Dans ce genre d'articles, ce n'est pas l'art qui est analysé, c'est la figure du poète à laquelle notre époque reste étonnamment attachée qui est célébrée. On aime que les poètes défendent la poésie, on leur reproche presque d'écrire des vers.

Les revues spécialisées, les colloques continuent - avec quelles difficultés, on le sait. Mais il y a aujourd'hui, en France et en Europe, des critiques de poésie de très grande 
qualité, capables d'analyser la création contemporaine. Ils parlent aux poètes. Ils sont souvent poètes. C'était le constat de Berardinelli en 1975 - le public de la poésie, c'est les poètes ${ }^{43}$.

Je prends un exemple pour faire saisir l'enjeu : Jean-Patrice Courtois publie en 2017 ses Théorèmes de la nature chez Nous ${ }^{44}$. C'est un livre très important qui inaugure un triptyque de poèmes consacrés à l'écologie. Il fera date.

Courtois est devenu un spécialiste de l'écologie : il collectionne les faits, il construit une bibliographie personnelle sur la question, il réfléchit à la question avec toutes sortes d'instruments. Tout cela est capital, mais l'essentiel n'est pas là. L'essentiel, c'est que ses textes sont des poèmes. La question de la description est alors urgente : que devient le contenu documentaire dans la forme poème? Comment inventer une phrase poétique pour évoquer la « destruction de la nature »?

Mathilde Vischer : Vous animez des ateliers de lecture et d'écriture dans les prisons : en quoi les échanges que vous avez avec les détenus nourrissent-ils votre propre travail poétique et/ou critique?

Martin Rueff: Tout comme Bacon se réjouissait que la photographie ait permis à la peinture de marquer sa différence - inventer un travail du visible -, de la même manière, la société de communication permet de marquer la différence des arts de la parole et de leur enseignement en dramatisant la question de l'adresse.

Qu'est-ce qu'un bon enseignant? C'est quelqu'un qui adresse un savoir sous la forme d'une parole destinée (c'est pourquoi il n'est pas question de faire des petits clips où un professeur singerait un cours devant une caméra - un cours c'est un événement de parole adressé, c'est tout : c'est peu, mais c'est énorme).

Et ce que le poème a de très singulier, n'est-ce pas qu'il s'adresse très précisément à une personne et qu'il touche plusieurs lecteurs (peu importe ici le nombre)? C'est parce qu'il invente une adresse particulière telle que je puisse le prendre pour moi, sur moi.

Avec des détenus, la question de l'adresse est décisive. On va en prison pour parler à des personnes et les convaincre qu'elles ont aussi le pouvoir de s'adresser. Oui : qu'elles peuvent se dresser pour s'adresser. C'est le contraire du redressement. Cette expérience est douloureuse, impose la pudeur, exige le respect. Quand on sort de prison, on a envie de se taire.

Mathilde Vischer: Vous avez, je crois, des livres de poèmes en préparation. Comment s'inscrivent-ils dans votre projet d'écriture et dans votre double appartenance au français et à l'italien?

Martin Rueff : a) Je ne saurais donner meilleure définition de mon travail que celle proposée par William Carlos Williams pour Paterson (qui reste pour moi un modèle indépassable) : «A poem is a complete little universe. It exists separately ${ }^{45}$ ». Un petit univers portatif - un habitacle sensible de mots que le lecteur est invité à visiter. Il faut organiser la visite, sans trop flécher le parcours. 
Je puis évoquer le livre de poèmes, La Jonction, que je viens d'achever (trois autres sont en travaux) et de donner à un éditeur : le titre désigne un nom de lieu mais aussi un dispositif pour faire converger deux langues dans le poème, entre «L'amer fait peau neuve » et « Noms de chiens (l'enrouement d'Actéon)».

"L'amer fait peau neuve » est une rêverie concertée sur le bleu et les bleus - le bleu du manteau de Marie qui a été utilisé pour le drapeau européen (l'affaire est insensée) devient le bleu de la mer méditerranéenne où s'achève un destin de l'Europe. Comme Pétrarque adresse à la fin du Canzoniere une prière à Marie j'en reprends l'élan. C'est une prière laïque. Mais le poème dérive vers une autre forme de bleus - les hématomes et c'est bien le fond de l'affaire - l'hématopoïétique. J'ai été frappé par ce terme technique (qui désigne en hématologie la formation du sang), et j'en ai fait un terme pour ma poétique. La formation des bleus devient une allégorie de la formation (naturelle) des poèmes. Je poursuis ce motif avec les hémophiles - la fin du livre peut rappeler Icare peut-être : un poème sur des fils qui meurent victimes de la folie des pères (j'ai en préparation un ensemble sur les filles). Ici encore des traditions hétérogènes viennent densifier le sang du poème.

« Noms de chiens (l'enrouement d'Actéon) » répondait à une commande du Musée de la Chasse et de la Nature et s'inscrivait en marge d'un vaste projet en cours sur les bestiaires (un imposant recueil que je construis depuis près de dix ans).

J'ai repris le mythe d'Actéon (ainsi que la tradition de ses interprétations) et j'ai travaillé dans deux directions. (Ces deux directions bien sûr n'étaient pas concertées au départ - je me lance, je suis des pistes, et c'est au moment des reprises, des infinies reprises que se dégagent des intelligibilités dont certaines se lèvent à l'insu du projet initial.)

D'une part j'ai « inventé » que c'est la nymphe Écho (figure singulière de la voix) qui avait manigancé l'arrivée d'Actéon dans le bois de Diane (un lointain modèle de cette structure est l'Hermès de Rilke qui convoie Orphée et Eurydice).

D'autre part, et surtout, je me suis concentré sur la liste des noms des chiens d'Actéon - une meute de quarante clébards nommés un par un par Ovide.

Après une ouverture consacrée à l'enrouement constitutif de l'énonciation poétique, je me tourne vers chaque figure du mythe et chacune a droit à son poème. C'est aussi un poème contre le devenir animal (animalier, mais aussi animaliste) d'une certaine poétique contemporaine. La voix animale est une possibilité du langage poétique, une fiction sensible qui est aussi une rêverie des limites. Je ne parle pas chien, je ne parle pas aux chiens. Le chien parle dans la voix des poèmes. C'est un peu autre chose. Le poème est aussi un poème critique.

C'est un nouveau livre de poèmes écrits par un écrivain en vers qui croit aux chances de la poésie.

b) Quant à la double appartenance, elle m'inspire cette réflexion sur le bilinguisme. Je l'expose dans sa version historico-théorique et je la reprends à mon compte.

Le $\mathrm{Xx}^{\mathrm{e}}$ siècle a cherché par plus d'une voie à déloger le sujet dans la langue (ce qui ne veut pas dire, comme on le voudrait hâtivement de la langue). Et par là, sans doute, cherche-t-il une voie pour l'insubordination, une manière de lutter contre la maîtrise, l'autorité, la posture du poète au verbe haut. Traduire ce n'est pourtant pas trahir, 
c'est servir : c'est honorer le conflit des fidélités qui anime chacun et auquel le poète, peut-être plus qu'aucun autre, voudrait être sensible, c'est vivre la double appartenance comme un double arrachement. C'est pour cela sans doute que le poète-traducteur Bernard Simeone oppose transmission et communication. Traduire, écrire dans deux langues, c'est faire œuvre en désœuvrant la possibilité de son œuvre. Le jour est arrivé où l'on peut faire la part de ces méthodes poétiques: la polyphonie, l'anonymat, l'hétéronymie ${ }^{46}$.

« Depuis 1945, écrit Samuel Beckett dans une lettre adressée à Hans Naumann le 17 février 1954, je n'écris plus en français. Pourquoi ce changement? Il ne fut pas raisonné. [...] Je vous donnerai quand même une piste : le besoin d'être mal armé ${ }^{47}$ ». $\mathrm{Au}$-delà du jeu de mots bien visible et d'une référence à Mallarmé qui n'a rien de fortuite, Beckett indique une nécessité, un besoin paradoxal. Il ne s'agit pas de s'armer avec de bonnes armes, il ne s'agit pas de ne pas s'armer, il s'agit de s'armer mal, de recourir à des outils inappropriés - à une « disappropriata maniera ». Beckett s'est expliqué sur ce besoin dans un entretien décisif où il redit une leçon de Hegel : "le contenu de l'idée de chaque époque trouve toujours sa forme convenable et adéquate, et c'est là ce que nous appelons les formes particulières de l'art ${ }^{48}$ ». Or voici ce que Beckett confie à Tom Driver :

Elle [la confusion] est tout autour de nous et nous n'avons qu'une seule possibilité désormais : la laisser rentrer. L'unique chance de rénovation désormais est d'ouvrir nos yeux et de voir le gâchis [waste]. C'est le genre de gâchis à partir duquel vous ne pouvez pas construire un sens.

Nous ne pouvons plus écarter le gâchis, parce que nous sommes parvenus à une époque où il a envahi notre expérience à tout instant. Il est là, il faut l'admettre. [...] Ce que je dis là ne signifie pas que l'art n'aura plus désormais de forme. Cela signifie uniquement qu'il aura une forme nouvelle; telle qu'il admette le chaos et ne prétende pas que le chaos lui est étranger. Forme et chaos restent distincts. Le chaos n'est pas réduit à la forme. C'est pourquoi la forme elle-même devient une préoccupation, parce qu'elle existe en tant que problème distinct de ce qu'elle exprime. Trouver une forme qui exprime le gâchis, telle est la tâche de l'artiste ${ }^{49}$.

Beckett propose un diagnostic de l'époque: le gâchis (mess). Ce gâchis pose un problème à l'artiste et ce problème devient sa mission : trouver une forme qui ne soit pas « étrangère » au chaos. Toute son œuvre tend à résoudre ce problème. Soulignons la thèse de Beckett : le gâchis est un contenu si informe qu'il est exclu que la forme puisse le relever, pour reprendre un terme de la dialectique spéculative.

Il faut le redire, ce problème est hégélien : «si l'on rappelle ce que nous avons déjà posé à propos du concept de beau et de l'art, deux éléments se présentent : d'un côté un contenu, une fin, une signification [ici le gâchis], de l'autre, l'expression, l'apparence et la réalité de ce contenu ; et en troisième lieu le rapport nécessaire en vertu duquel les deux termes se pénètrent réciproquement, à un point tel que l'extérieur, le particulier, n'apparaît plus que comme manifestation de l'extérieur. L'œuvre d'art ne présente que ce qui a une relation essentielle avec le contenu et l'exprime ${ }^{50} »$.

Or précisément, la traduction, ou l'écriture dans une langue autre qui ne serait ni tout à fait la langue maternelle, ni tout à fait une autre, ont pu apparaître pour certaines et certains comme la solution formelle au problème posé par l'époque. Il ne s'agit plus alors de rendre la langue élastique sans la déchirer : il s'agit d'emprunter une autre langue pour se confronter à des déchirures qui ne sauraient plus se dire 
dans une langue d'appartenance, mais qui doit porter comme matériellement les traces de la déchirure. C'est le cas de Beckett, qui se force à écrire dans une langue qui n'est pas la sienne. On pensera au modèle offert par les peintures gauches et graciles de Cy Twombly: les écritures qui les traversent, écrit Barthes, sont les «bribes d'une paresse, donc d'une élégance extrême». Barthes comparait cette décision au satori des Japonais: "par une circonstance infime, voire dérisoire, aberrante, voire farfelue, le sujet s'éveille à une négativité radicale (qui n'est plus une négation $)^{51}$ ». Cette négativité radicale se confond chez Twombly avec la décision la plus grave pour un peintre - celle du gauche :

Que ces graphismes, ces compositions soient comme "gauches", cela renvoie TW au cercle des exclus, des marginaux - où il se retrouve bien entendu, avec les enfants, les infirmes: le "gauche » (ou le " gaucher ») est une sorte d'aveugle : il ne voit pas bien la direction, la portée de ses gestes; sa main seule le guide, le désir de sa main, non son aptitude instrumentale; l'œil, c'est la raison, l'évidence, l'empirisme, la vraisemblance, tout ce qui sert à contrôler, à coordonner, à imiter, et comme art exclusif de la vision, toute notre peinture passée s'est trouvée assujettie à une rationalité répressive. D'une certaine façon, Twombly libère la peinture de la vision, car le "gauche » (le « gaucher ») défait le lien de la main et de l'œil : il dessine sans lumière (ainsi faisait Twombly, à l'armée).

Tout comme pour Twombly, dessiner de la main gauche, c'est se laisser échapper pour se désapproprier, de la même manière, traduire, ce fut, au $\mathrm{Xx}^{\mathrm{e}}$ siècle, une arme choisie pour se laisser échapper, pour se désapproprier. Ce fut le cas de Paul Celan, mais aussi de cette extraordinaire poétesse-traductrice que fut Amelia Rosselli. Surplombe, pourtant, dans sa fragilité, gaucher se contrariant lui-même, prince des cassés, et entre tous, le mieux mal armé, Antonin Artaud.

c) On peut écrire en deux langues pour gagner en force expressive, on peut écrire en deux langues pour perdre en force expressive. On a donc affaire à deux modèles qui sont vécus dans l'histoire des hommes avant d'être élaborés dans la théorie. Dans un cas comme dans l'autre le propos est énergétique : faire d'une force (ah vous parlez deux langues... quelle chance) une faiblesse, faire d'une faiblesse (ah mais vous n'habitez plus nulle part) une force.

Cette affaire de poétique rejoint un enjeu politique contemporain considérable que résume une formule de Bruno Latour dans un livre bouleversant: "la nouvelle universalité, c'est de sentir que le sol est en train de céder ${ }^{52}$ ».

J'ai été frappé récemment de voir que Caillois achevait Obliques par cette remarque philologique : «j'ai lu - ou croyant me souvenir, j'invente - qu'il existe des langues où le même verbe signifie "tomber (dans un piège)" et "réussir (trouver son accomplissement)" ${ }^{\prime 3} "$.

C'est la morale d'Icare le bilingue : drôle d'amphibien de la full immersion, le bilingue a deux fois plus de chances de se noyer. 


\section{NOTES}

1. E. Montale, L'Opera in versi, Turin, Einaudi, 1980, p. 28.

2. La revue, trimestrielle, a été fondée en 1977 par M. Deguy.

3. Les premières traductions sont parues respectivement en 1955 (trad. J. Bertrand, Gallimard), 1960 (trad. J. Bertrand, Seuil) et 1962 (trad. M. Javion, Seuil).

4. Pour Berman, ce potentiel peut répondre au critère suivant : « le rapport interne qu'une œuvre entretient avec la traduction (ce qu'elle contient en soi de traduction ou de non-traduction) détermine idéalement son mode de traduction interlangues, ainsi que les "problèmes" de traduction qu'elle peut poser » (A. Berman, La Traduction et la lettre, ou l'auberge du lointain, [1985], Paris, Le Seuil, 1999, p. 113).

5. A. Berman, Pour une critique des traductions, Paris, Gallimard, 1995.

6. J. -P. Courtois (dir.), De la retraduction : Le cas des romans, Bruxelles, La lettre volée, 2014.

7. On pourrait à ce propos rappeler le livre important de J. -P. Seguin, L'Invention de la phrase au XVIII ${ }^{\mathrm{e}}$ siècle, Louvain, Peeters, 1993.

8. « Entretien avec Martin Rueff», Po\&sie, $n^{\circ} 120,2007$, p. 292.

9. «Ce n'est pas pour moi », « vraiment pas mon genre » pour citer Swann.

10. F. Pejoska Bouchereau, P. Marillaud et R. Gauthier (dir.) «Le roman de l'étrangéisation », dans Écritures évolutives, colloque d'Albi «Langages et signification», Université Toulouse Le Mirail, 2010, p. 265-278. Disponible en ligne: <https://issuu.com/walterap/docs/cals2010> (consulté le 21/01/19).

11. Voir entre autres M. Foucault, «L'écriture de soi », Corps écrit, $n^{\circ} 5,1983$, p. 3-23 (repris dans le tome IV des Dits et Écrits, texte ${ }^{\circ} 329$, Paris, Gallimard, 2001).

12. G. Deleuze, Deux régimes de fous et autres textes (1975-1995), Paris, Minuit, coll. «Paradoxe», 2003, p. 326-328.

13. «Je me propose de dire les métamorphoses des formes en des corps nouveaux» (Ovide, Les Métamorphoses, I, 1-2, G. Lafaye et J.-P. Néraudau (trad.), Paris, Gallimard, 1992, coll. «Folio Classique », p. 42).

14. J. Keats, Lettres à Fanny et autres correspondances, R. Davreu et Cl. Mouchard (trad.), Paris, Belin, 1993, p. 207.

15. On prendra la mesure de cette question en lisant l'essai que M. Vischer a consacré à deux poètes-traducteurs : P. Jaccottet et F. Pusterla dans La Traduction, du style vers la poétique, Philippe Jaccottet et Fabio Pusterla en dialogue, Paris, Kimé, 2009.

16. B. Simeone, Écrire, traduire, en métamorphose : L'Atelier infini, Paris, Verdier, 2014, p. 11.

17. L. Althusser, Pour Marx, Paris, Maspero, coll. « Théorie », 1968.

18. E. Kant, "Théorie et pratique", dans Théorie et pratique, D’un prétendu droit de mentir par humanité, La Fin de toutes choses et autres textes, Fr. Proust (trad.), Paris, GF-Flammarion, 1994.

19. Jeudi 6 octobre 2016, «Leysin, les autres - trois remarques sur la traduction poétique », CUSO, Leysin, conférence non publiée.

20. Le Cantique des cantiques, E. Renan (trad.), [1860], Paris, Arléa, 1991, p. 13.

21. G. Bassani, Le Jardin des Finzi Contini, M. Arnaud (trad.), Paris, Gallimard, 1975.

22. Bassani possédait la traduction des poèmes d'E. Dickinson due à M. Bini, Milan Denti, 1949. Voici la version anglaise de ce poème de 1862 (J 449 ou F 448) :

«I died for Beauty - but was scarce / Adjusted in the Tomb / When One who died for Truth, was lain / In an adjoining Room - / He questioned softly "Why I failed?" / "For Beauty", I replied - / "And I - for Truth - Themself are One / - We Brethren, are", He said - / And so, as Kinsmen, met a Night - / We talked between the Rooms - / Until the Moss had reached our lips - / And covered up - our names - ». 
23. La traduction française est celle que propose, en note, M. Arnaud, dans Le Jardin des FinziContini, ouvr. cité.

24. C. Pavese, Travailler fatigue, La Mort viendra et elle aura tes yeux, Poésies variées, G. de Van (trad.), Paris, Gallimard, 1979.

25. « Guadagnare nei cambi. Il fine del poeta-traduttore », S. Picciaiola (trad.), Testo a fronte, $\mathrm{n}^{\circ} 49$, $2^{\text {nd }}$ semestre 2013, p. 33.

26. P. Valéry, « Naissance de Vénus » dans Euvres I, Paris, Gallimard, coll. «Bibliothèque de la Pléiade ", 1957, p. 77.

27. M. Deguy, La poésie n'est pas seule : Court traité de poétique, Paris, Seuil, 1988.

28. Po\&sie, $\mathrm{n}^{\circ} 109$ et 110, « 30 ans de poésie italienne, 1975-2004», Paris, Belin/Humensis, 2004.

29. Par quoi il entend à la fois le polyglottisme et la variété des styles et des genres. On songe bien sûr à Bakhtine, que Contini ne connaissait pas alors.

30. J. Rancière, Mallarmé o la politica della sirena, A. Serra (trad.), Bologna, Clueb, 2000.

31. S. Junod, Agrippa d'Aubigné ou les misères du prophète, Genève, Droz, 2008.

32. M. Rueff, « Blood Shift », dans Comme si quelque : Livre de poésie, Chambéry, Comp'act, coll. « La polygraphe », 2006, p. 204.

33. Voir le blog du poète L. André qui associe également poésie et blood shift en faisant référence à J. Mayol (Homo Delphinus, Grenoble, Glénat, 1986). En ligne : <http://lionelandre.blogspot.com/ 2016_04_16_archive.html> (consulté le 24 avril 2019).

34. R. Char, "Alliés substantiels ", dans Recherche de la base et du sommet [1955], Euvres complètes, Paris, Gallimard, coll. «Bibliothèque de la Pléiade », 1995, p. 671-708.

35. E. Kant, Critique de la faculté de juger [1790], A. Renaut (trad.), Paris, Flammarion, coll. «GFPhilosophie ", §49, AK V, 318.

36. M. Rueff, «L'Intraduisible, même ", dans Après Babel, traduire, B. Cassin (dir.), Arles/Marseille, Actes Sud/Mucem, 2016, p. 232.

37. A. Artaud, « Onze lettres à Anaïs Nin », Lettre du 3 avril 1933, Tel Quel, $n^{\circ} 20$, Paris, Seuil, hiver 1965, p. 4.

38. A. Artaud, "Avertissement au Moine", dans CEuvres, Le Moine raconté par Antonin Artaud, Gallimard, coll. « Quarto », 1966, p. 3519.

39. P. Celan, « Le Méridien », Le Méridien et autres proses, J. Launay (trad.), Paris, Seuil, 2002, p. 72.

40. E. Benveniste, Baudelaire, Limoges, Lambert-Lucas, 2011.

41. Propos cités par J. Scherer, Grammaire de Mallarmé, Paris, Nizet, 1977, p. 41-42.

42. O. Emil'evič Mandel'štam, Euvres complètes, J.-C. Schneider (trad.), Paris/Genève, Le Bruit du temps/La Dogana, 2018.

43. A. Berardinelli, F. Cordelli, Il pubblico della poesia [1975], Rome, Castelvecchi, coll. « Le Navi », 2015.

44. J.-P. Courtois, Théorèmes de la nature, Caen, Nous, coll. « Disparate », 2017.

45. W. Carlos Williams, Paterson, New York, New Directions, 1995, p. 224-225.

46. Avec Pessoa, chaque hétéronyme nomme certes une virtualité poétique du même poète. Mais une telle expression est trompeuse, car elle laisserait supposer qu'un moi, disons un Je synthétique, mettrait en scène les quatre hétéronymes de Pessoa pour s'exprimer à travers eux. C'est plutôt l'inverse qui est vrai. L'hétéronymie rend difficile la simple distinction de l'auteur comme individu réel à l'extérieur du texte et de la fonction-auteur comme principe d'attribution interne : car c'est de l'intérieur des poèmes que naissent leurs auteurs - les hétéronymes. Voir Rueff Martin, «Vitesse de Risset», Po\&sie, 2014/3, n 149-150, p. 14-19. DOI : 10.3917/poesi. 149.0014 .

47. The Letters of Samuel Beckett (1941-1956), G. Graig, M. Dow Fehsenfeld, L. More Overbeck et D. Gunn (dir.), Cambridge, C.U.P, vol. 2, 2011, p. 463-464. 
48. G. W. Fr. Hegel, Esthétique I, Paris, Le Livre de Poche, 2003, p. 400. Ainsi, non seulement les contenus de l'art et ses formes sont historiques, mais leur dialectique aussi - voir p. 130, p. 160, p. 167-168 et p. 419.

49. " It is all around us and our only possibility now is to let it in. The only chance of renovation is to open our eyes and see the mess. It is not a mess you can make sense of. [...] How could the mess be admitted, because it appears to be the very opposite of form and therefore destructive of the very thing that art ought itself to be? But now we can keep it out no longer because we have come into a time when "it invades our experience at every moment. It is there and it must be allowed in" [...] What I am saying does not mean that there will henceforth be no form in art. It only means that there will be new form, and that this form will be of such a type that it admits the chaos and does not try to say that the chaos is really something else. The form and the chaos remain separate. The latter is not reduced to the former. That is why the form itself becomes a preoccupation, because it exists as a problem separate from the material it accommodates. To find a form that accommodates the mess, that is the task of the artist now » (S. Beckett, interview with T. Driver, in Columbia University Forum, Summer, 1961, p.21-25, dans Samuel Beckett: The Critical Heritage, L. Grover et R. Federman (dir.), London, Routledge and Kegan Paul, 1979, p. 219). 50. G. W. Fr. Hegel, Esthétique, ouvr. cité., p. 160-161.

51. R. Barthes, « Non multa sed multum », dans Y. Lambert (dir.), Catalogue raisonné des œuvres sur papier de Cy Twombly, vol. VI, 1973-1976, Milan, Multipha, 1979, p. 7-13 [rééd. dans L'Obvie et l'obtus, Paris, Seuil, 1982, p. 145-162].

52. B. Latour, Où atterrir ?, Paris, La Découverte, 2018, p. 19.

53. R. Caillois, Obliques [1975], Paris, Gallimard, 1987, p. 256.

\section{RÉSUMÉS}

Dans cet entretien avec Mathilde Vischer, Martin Rueff s'exprime sur son rapport aux langues, à la poésie et au roman, ainsi que sur sa double activité de poète et de traducteur. Il explore le nouage entre écriture et traduction et met en évidence la dimension hautement esthétique et poétique de l'acte traductif. Après avoir évoqué ses retraductions de la trilogie des ancêtres de Calvino, Martin Rueff explique en quoi la traduction de poèmes est pour lui une pratique de désubjectivation, intimement dépendante des théories sous-jacentes qui influencent tout choix traductif. Il évoque également sa conception de la traduction poétique comme une tentative de reproduire non pas ce que le poème " fait dans sa langue de départ », mais plutôt ce qu'il « fait $a$ » cette langue. Il réfléchit ainsi aux spécificités du langage poétique et s'interroge finalement sur la dimension plurilingue de son œuvre, à l'instar d'Icare crie dans un ciel de craie (Paris, Belin, 2007), où Icare traversé, dans sa chute, par les langues et les poèmes de ses pères, les traverse et construit ce faisant la forme du poème. L'auteur accepte de révéler ici quelques enjeux de son livre de poésie La Jonction (Caen, Nous, 2019). Martin Rueff nous invite à nous placer dans une disponibilité particulière à l'égard des textes et de la langue, cette « écoute flottante » qui permet au poète et au traducteur d'entrer au cœur d'une expérience donnant à la langue la possibilité de porter les traces de sa propre déchirure.

In this interview with Mathilde Vischer, Martin Rueff speaks of his relationship with languages, and how he conceives of his own writing and translation practices. He explores the profound connections shared by these two activities, bringing to light the highly aesthetic and poetic 
dimension of the translational act. Rueff first refers to his retranslations of Calvino's trilogy, Our Ancestors, then explains how translating poems is for him a process of desubjectification, intimately tied to underlying theories that affect each translational choice. He perceives poetic translation as an attempt to reproduce not what the poem "does in the source language", but what it "does to" the language. He thereby probes the specificities of poetic language and considers the plurilingual aspect of his work, like Icare crie dans un ciel de craie (Belin, 2007), where Icarus crossed, in his fall, by the languages and poems of his fathers, crosses them and thus builds the shape of the poem. He reveals some of the issues in his poetry book La Jonction (Nous, June 2019). Martin Rueff invites us to place ourselves in a particular availability with regard to texts and language, this "floating listening" that allows the poet and translator to enter into the heart of an experience that gives the language the possibility to bear the traces of its own tearing.

\section{AUTEURS}

\section{MARTIN RUEFF}

Martin Rueff vit entre le français et l'italien, il traduit - essentiellement - de l'italien, écrit en français, mais la langue italienne s'invite souvent dans ses textes. Interrogé ici en tant que poète et traducteur, il est aussi essayiste, spécialiste de Rousseau et de littérature italienne, virtuose des liens entre philosophie et littérature, entre poésie et poétiques contemporaines. Il a été responsable de nombreuses éditions critiques (Foucault, Lévi-Strauss, Pavese, Starobinski), il œuvre comme directeur de la collection « Terra d'altri » chez Verdier, est co-rédacteur en chef de la revue Po\&sie et professeur à l'Université de Genève. Comme traducteur de poésie et de romans d'auteurs italiens, il a signé les traductions de nombreux poèmes du double volume de Po\&sie 109-110 consacré à 30 ans de poésie italienne qu'il a dirigé, mais aussi, notamment, de poèmes de Zanzotto et De Signoribus, ainsi que des retraductions de plusieurs romans de Calvino. Il a également traduit de nombreux essais philosophiques, avec une fidélité particulière à Agamben et Ginzburg.

\section{MATHILDE VISCHER}

Mathilde Vischer est traductrice littéraire et professeure associée à la Faculté de traduction et d'interprétation de l'Université de Genève. Elle a traduit des poètes contemporains (Felix Philipp Ingold, Fabio Pusterla, Alberto Nessi, Pierre Lepori, Massimo Gezzi, Elena Jurissevich, Leopoldo Lonati) et publié des articles et des essais portant sur la poésie et sur la traduction (Philippe Jaccottet traducteur et poète, une esthétique de l'effacement, Cahiers du Centre de traduction littéraire $n^{\circ}$ 43, Lausanne, 2003 et La Traduction, du style vers la poétique : Philippe Jaccottet et Fabio Pusterla en dialogue, Paris, éditions Kimé, 2009). Elle a également publié un livre de poèmes, Lisières (Dijon, p.i.sage intérieur, 2014 ; Prix du poème en prose Louis Guillaume 2015 et Prix Terra Nova 2015). 